\title{
Choroidal Nevus in an Eye with Polypoidal Choroidal Vasculopathy
}

\author{
Kazunobu Asao Noriyasu Hashida Kohji Nishida \\ Department of Ophthalmology, Osaka University Graduate School of Medicine, \\ Osaka, Japan
}

\section{Key Words}

Aflibercept · Autofluorescence · Choroidal nevus · Pigment epithelial detachment · Polypoidal choroidal vasculopathy

\begin{abstract}
Purpose: To report an eye with polypoidal choroidal vasculopathy (PCV) and a choroidal nevus. Methods: This is an observational case report. Results: A healthy 69-year-old woman was referred to the Osaka University Hospital with a diagnosis of a macular tumor. She complained of having distorted vision in her left eye. The medical history of the patient was unremarkable. At the initial examination, her best-corrected visual acuity (BCVA) was 20/20 in both eyes, and the intraocular pressure was $18 \mathrm{~mm} \mathrm{Hg}$ in both eyes. A slit-lamp examination showed no abnormalities in the anterior segment of both eyes and a fundus examination of the left eye showed a slightly elevated juxtafoveal chorioretinal lesion and polyp-like reddishorange lesions. The juxtafoveal choroidal lesion was located beneath a choroidal neovascularization (CNV). An optical coherence tomography confirmed CNV with pigment epithelial detachment (PED). Fluorescein angiography showed juxtafoveal hyperfluorescence due to CNV. Indocyanine green angiography demonstrated a branching choroidal vascular network that resembled polypoidal lesions. A fundus autofluorescence showed a mosaic pattern and a slight hyperautofluorescence at the CNV. We diagnosed the patient as having PCV. Aflibercept was injected intravitreally because of her PED. After the injection, PED improved and her visual acuity remained stable during the 12-month follow-up period. Conclusions: In cases of PCV, FAF images are helpful in determining the status of the posterior pole. Intravitreal injections of aflibercept can improve PED associated with CNV, and the BCVA will remain stable for at least 12 months.


Asao et al.: Choroidal Nevus in an Eye with Polypoidal Choroidal Vasculopathy

\section{Introduction}

Choroidal nevi are the most common benign intraocular tumors of the eye, and their prevalence ranges from 4 to $8 \%$ of the population [1]. Only under $1 \%$ of patients $(n=322)$ have choroidal neovascularization (CNV) accompanying the nevus [1]. In eyes with a nevus, secondary retinal pigment epithelial changes such as drusen, atrophy, fibrous metaplasia, orange pigmentation, pigment epithelial detachment (PED) and subretinal fluid (SRF) can also be present [2]. However, a nevus accompanying polypoidal choroidal vasculopathy (PCV) is a rare complication [1].

On fundus examination, the most suggestive findings of PCV are orange-red lesions, and PCV is characterized by the presence of a branching vascular network in association with polypoidal choroidal vascular lesions that cause subretinal leakage, subretinal hemorrhage and PED [3]. Fifty-five percent of the Japanese patients with age-related macular degeneration (AMD) have PCV [3]. Anti-vascular endothelial growth factor (VEGF) injections are used to treat eyes with PCV to maintain or improve the visual acuity (VA) [4, 5]. Optical coherence tomography (OCT) and fundus autofluorescence (FAF) can be used to examine the morphological features of PCV and the choroidal nevus [2]. The FAF images are helpful in determining whether the lesion is a malignant tumor [6].

Some pigmented lesions such as choroidal melanoma, pigmented choroidal metastasis, subretinal or choroidal hemorrhage and retinal pigment epithelial tumors can transform into malignant melanomas. Therefore, it is important to exclude these tumors during the diagnostic period [7].

Intravitreal aflibercept injections have been reported to improve the functional and anatomical changes in the short term in subjects with active exudative AMD [5]. The aim of this study was to determine whether intravitreal aflibercept could successfully treat an eye with PCV associated with choroidal nevus.

\section{Case Report}

A healthy 69-year-old woman was referred to the Osaka University Hospital with a diagnosis of a macular tumor. She complained of distorted vision in her left eye. Her medical and family history was unremarkable. Before referral, there were no systemic workups for tuberculosis, sarcoidosis and toxoplasmosis. We excluded other diseases such as choroidal melanoma, pigmented choroidal metastasis and retinal pigment epithelial tumors by $\mathrm{N}$ isopropyl-p-[123I]-iodoamphetamine single photon emission CT, and her general condition was good.

At our initial examination, her best-corrected visual acuity was 20/20 in both eyes, and the intraocular pressure was $18 \mathrm{~mm} \mathrm{Hg}$ in both eyes. A slit-lamp examination showed no abnormalities in the anterior segment of either eye. An ophthalmoscopy of the left eye revealed a slightly elevated juxtafoveal chorioretinal lesion and polyp-like reddish-orange lesions (fig. 1a). There was also a PED around the lesion. In the macular area, there were no exudative changes such as hard exudates and macula edema.

Fluorescein angiography (TRC-50LX; Topcon, Tokyo, Japan) showed juxtafoveal hyperfluorescence due to the underlying CNV (fig. 1b). Indocyanine green angiography (Heidelberg Retinal Angiograph 2, Heidelberg, Germany) showed branching choroidal vascular networks (fig. 1c), and magnified images showed that the lesions resembled those from eyes with PCV (fig. 1d). The FAF images had a darker hypoautofluorescence than the surrounding 
areas and slight hyperautofluorescence suggesting an exudation from the CNV within the nevus (fig. 1e).

We also performed OCT examinations with 3 OCT instruments: a Heidelberg Spectralis (Cirrus HD-OCT; Carl Zeiss Meditec, Inc., Dublin, Calif., USA), a Heidelberg Spectralis HRA + OCT (Heidelberg Engineering), and a prototype high-penetration OCT Topcon). The OCT examinations confirmed the CNV with an overlying PED, and the PED maintained (fig. 2a). Because of the PED, aflibercept was injected intravitreally which improved the PED (fig. 2b). Her VA remained stable for the 12-month follow-up period.

The patient provided her written informed consent for the treatment. The study adhered to the tenets of the Declaration of Helsinki.

\section{Discussion}

We herein reported our findings in an eye with PCV accompanied by a choroidal nevus. A choroidal nevus associated with $\mathrm{CNV}$ is rare and may arise at extrafoveal or subfoveal areas [1]. With recent improvements of OCT and FAF, PCV lesions can be observed in greater detail. Thus, photoreceptor loss and decreased density have been detected in about one-half of the cases with choroidal nevus [2]. FAF was found to be useful in evaluating the retinal pigment epithelium (RPE) noninvasively, and our examination showed an orange-pigmented lesion, which is known to be a risk factor for malignant transformation [6]. The FAF features appeared not to be affected by the tumor thickness as reported for eyes with choroidal melanoma. The FAF images were also helpful in detecting the margins of the PCV. Our case showed that a nevus located near the macular region appeared as a dark hypoautofluorescent lesion, and the area outside the macular region had a mixed autofluorescence. At the PCV, a slight hyperautofluorescence was detected, although the vessels may have blocked the autofluorescence.

For patients with PCV, intravitreal anti-VEGF injections have been used to treat CNV [4]. If $\mathrm{CNV}$ is juxtafoveal and a choroidal nevus is present, intravitreal bevacizumab or ranibizumab has been shown to be an effective treatment $[8,9]$. However, in some eyes, bevacizumab and ranibizumab were not able to depress the CNV activity [5]. Then, a switch to aflibercept resulted in improved visual and anatomical outcomes.

The results of earlier studies showed that peripheral exudative hemorrhagic chorioretinopathy, congenital hypertrophy of the RPE, hemorrhagic PED, choroidal hemangioma, AMD and RPE hyperplasia present with lesions that resemble a choroidal nevus [2]. To reduce the risk of a decrease in VA, it might be necessary to prevent the exudative changes as soon as possible. In patients with recalcitrant AMD, intravitreal aflibercept injections led to an anatomic improvement at 12 months with a reduction in the proportion of eyes with subretinal fluid and reduced PED while preserving the VA [10]. In our case, aflibercept was injected to prevent the spread of subretinal fluid and PED near the macula, and also to reduce the risk of the choroidal nevus causing a decrease in the VA [7]. Our patient strongly requested to be treated as soon as possible. The aflibercept injection improved the exudative changes, and no adverse events were found. During the follow-up period, her BCVA remained stable at $20 / 20$.

There are some limitations to this study. This was a retrospective study of only 1 eye. However, the rarity of this combination makes it difficult to study more cases. The results showed that aflibercept is effective in the treatment of vision-threatening CNV overlying a choroidal nevus. Without exudative changes, it is difficult to justify treating an eye with intravitreal anti-VEGF agents. Additional prospective long-term studies are needed to 
Asao et al.: Choroidal Nevus in an Eye with Polypoidal Choroidal Vasculopathy

determine the etiology, clinical course, and VA of patients with choroidal nevus associated with PCV.

In conclusion, we present our findings in a case of a choroidal nevus associated with CNV in an eye with PCV. FAF images were helpful in determining the status of the CNV and nevus. Intravitreal injections of aflibercept led to a decrease in the exudative changes caused by CNV.

\section{References}

1 Li HK, Shields CL, Mashayekhi A, et al: Giant choroidal nevus clinical features and natural course in 322 cases. Ophthalmology 2010;117:324-333.

2 Materin MA, Raducu R, Bianciotto C, et al: Fundus autofluorescence and optical coherence tomography findings in choroidal melanocytic lesions. Middle East Afr J Ophthalmol 2010;17:201-206.

-3 Maruko I, Iida T, Saito M, et al: Clinical characteristics of exudative age-related macular degeneration in Japanese patients. Am J Ophthalmol 2007;144:15-22.

4 Chiang A, Bianciotto C, Maguire JI, et al: Intravitreal bevacizumab for choroidal neovascularization associated with choroidal nevus. Retina 2012;32:60-67.

5 Singh RP, Srivastava S, Ehlers JP, et al: A single-arm, investigator-initiated study of the efficacy, safety and tolerability of intravitreal aflibercept injection in subjects with exudative age-related macular degeneration, previously treated with ranibizumab or bevacizumab: 6-month interim analysis. Br J Ophthalmol 2014;98:22-27.

6 Chin K, Finger PT: Autofluorescence characteristics of suspicious choroidal nevi. Optometry 2009;80:126130.

7 Shields CL, Furuta M, Mashayekhi A, et al: Clinical spectrum of choroidal nevi based on age at presentation in 3,422 consecutive eyes. Ophthalmology 2008;115:546-552.

-8 Tuncer S, Tugal-Tutkun I: Choroidal neovascularization secondary to choroidal nevus simulating an inflammatory lesion. Indian J Ophthalmol 2013;61:305-306.

-9 Papastefanou VP, Nogueira V, Hay G, et al: Choroidal naevi complicated by choroidal neovascular membrane and outer retinal tabulation. Br J Ophthalmol 2013;97:1014-1019.

10 Grewal DS, Gill MK, Sarezky D, et al: Visual and anatomical outcomes following intravitreal aflibercept in eyes with recalcitrant neovascular age-related macular degeneration: 12-month results. Eye (Lond) 2014;28:895-899. 


\section{Case Reports in}

Ophthalmology

\begin{tabular}{l|l}
\hline Case Rep Ophthalmol 2014;5:463-467 \\
\hline DOI: 10.1159/000370044 & $\begin{array}{l}\text { ○ 2014 S. Karger AG, Basel } \\
\text { www.karger.com/cop }\end{array}$ \\
\hline
\end{tabular}

Asao et al.: Choroidal Nevus in an Eye with Polypoidal Choroidal Vasculopathy
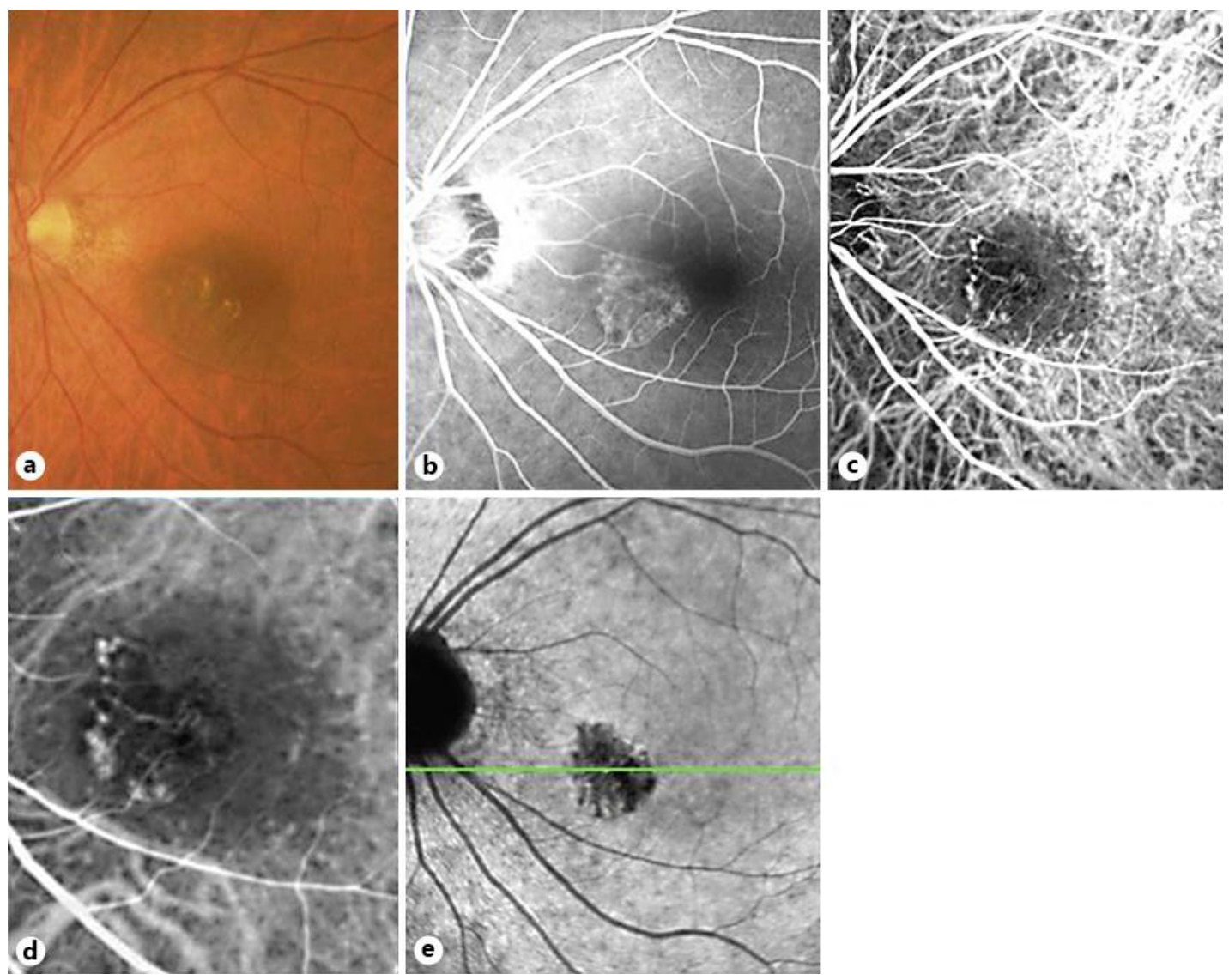

Fig. 1. a Fundus photograph of a 69-year-old woman with a juxtafoveal choroidal nevus. b Fluorescein angiography showing a juxtafoveal hyperfluorescence due to CNV. c, d Indocyanine green angiography showing a network of vessels nasal to the fovea. e FAF image showing that the nevus is located closer to the macular region (as shown by the darker hypoautofluorescence) than outside the macular region. A slight hyperautofluorescence is detected at the PCV.

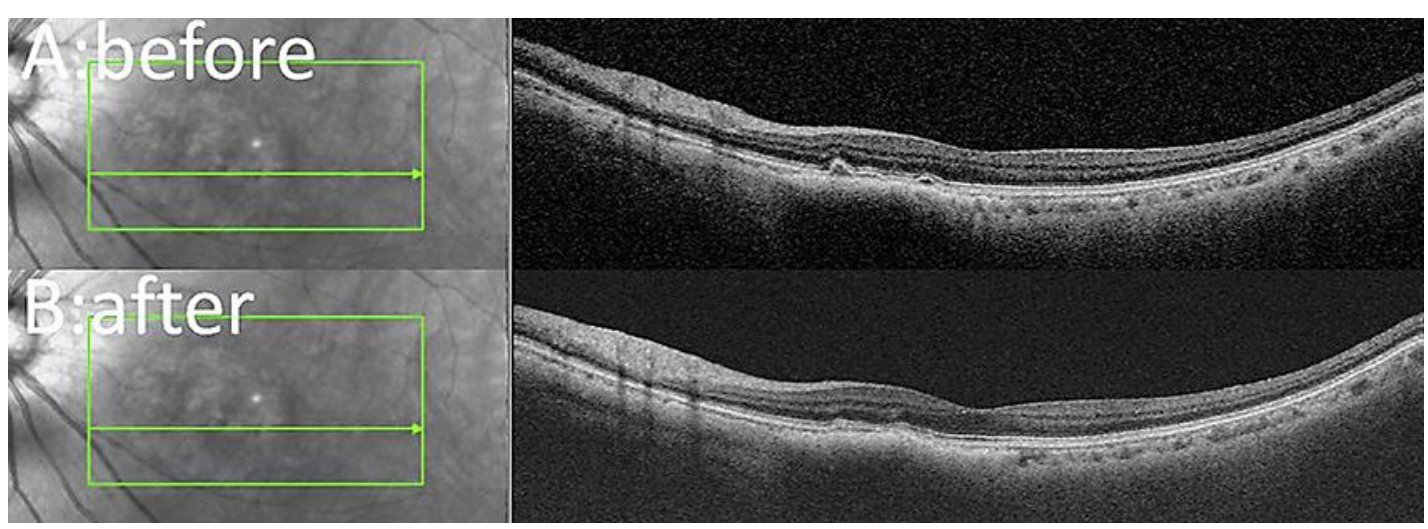

Fig. 2. A OCT confirms the presence of CNV with slight PED before the aflibercept injection. B The CNV is reduced after aflibercept injection. 